\title{
Carnets
}

Revue électronique d'études françaises de l'APEF

Première Série - 2 Numéro Spécial | 2010

Littératures nationales: suite ou fin. Résistances, mutations \& lignes de fuite

\section{Entre le réel et l'insolite : l'image du Maroc contemporain dans la prose de Tahar Ben Jelloun entre 1994 et 2009}

\section{Magdalena Zdrada-Cok}

\section{(2) OpenEdition}

\section{Journals}

Édition électronique

URL : http://journals.openedition.org/carnets/4898

DOI : $10.4000 /$ carnets.4898

ISSN : 1646-7698

Éditeur

APEF

Édition imprimée

Date de publication : 1 juin 2010

Pagination : 43-53

\section{Référence électronique}

Magdalena Zdrada-Cok, «Entre le réel et l'insolite : l'image du Maroc contemporain dans la prose de Tahar Ben Jelloun entre 1994 et 2009 », Carnets [En ligne], Première Série - 2 Numéro Spécial | 2010, mis en ligne le 16 juin 2018, consulté le 19 avril 2019. URL : http://journals.openedition.org/ carnets/4898; DOl : 10.4000/carnets.4898

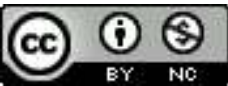

Carnets est mis à disposition selon les termes de la licence Creative Commons - Atribution - Pas d'utilisation commerciale 4.0 International. 


\title{
ENTRE LE REEL ET L'INSOLITE \\ L'image du Maroc contemporain dans la prose de Tahar Ben Jelloun entre 1994 et 2009
}

MAGDALENA ZDRADA-COK

Université de Silésie (Pologne)

cok@onet.pl

\begin{abstract}
Résumé
L'étude se concentre sur l'analyse de L'homme rompu (1994), Partir (2006) et Au pays (2009). Résultats d'un mariage du romanesque avec la sociologie et le journalisme, fruit des réflexions de l'auteur sur la conception de la littérature - monde en français, ses trois romans réalisent surtout la fonction mimétique de la littérature, en rompant avec l'autotélisme et la subversion formelle propres aux écrits benjellouniens antérieurs. Présents dans les dénouements des textes, les motifs fantastiques fonctionnent comme une sorte de commentaire de la part de l'auteur - qui, généralement, en tant qu'écrivain public - préfère donner la parole à ses personnages - prototypes des trois générations des Marocains. L'article étudie surtout la catégorie de la "patrie" qui sert dans les romans à véhiculer les problèmes socio-politiques du Maroc actuel.
\end{abstract}

\begin{abstract}
The article is dedicated to three novels by Tahar Ben Jelloun: L'Homme rompu (1994), Partir (2006) and Au pays (2009).Being a result of the author's sociological and journalistic interests, and his reflection on la littérature-monde en français, these novels perform the mimetic function of literature. Contrast between the rare fantasy threads and the dominant realism makes the author's particular comment. The object of study is the traditional category of "homeland", and that's the way Ben Jelloun's novels present the sociological and political matters of the contemporary Morocco.
\end{abstract}

Mots-clés: Roman sociologique, réalisme et fantastique, Patrie

Keywords: Sociological novel, Realism and fantasy, Homeland 
II existe dans l'écriture benjellounienne une tension entre l'engagement et la rupture avec le réel. Ces deux désirs opposés s'inscrivent déjà dans la vocation littéraire de l'écrivain qui se perçoit - en tant qu'artiste - comme un être de déchirement:

Je crois à l'histoire du double: ainsi je serais habité par quelqu'un d'autre - pas forcément sympathique - dont j'aurais les gestes et pas la mémoire, quelqu'un qui se serait glissé en moi à mon insu et qui vivrait un peu de sa vie et un peu de la mienne. Plein de cette présence qui me trahit (Ben Jelloun, 1983: 128).

En effet, déclenché par une révolte, son acte d'écrire traduit un besoin d'agir et d'en parler, mais, en même temps, il reste inséparable d'une volonté de fuir dans un monde pantextuel, dans une réalité parallèle: "écrire au lieu de vivre" (ibid:: 160). D'abord, dans deux camps disciplinaires à El Hajeb et à Ahermemou dans l'Est du Maroc, puis à Paris, où il découvre le monde de l'immigration ouvrière maghrébine, Ben Jelloun se doit d'abord d'assumer un rôle d'"écrivain public" pour parler au nom de ses compatriotes privés de parole. "Mes premières phrases ont surgi d'une blessure. [...] J'écrivis L'Aube des dalles dans la fébrilité du corps oppressé" - avoue-t-il dans son essai autobiographique (ibid:: 109). Mais déjà à l'époque où la littérature devient son lieu de combat, elle promet au jeune artiste d'échapper au réel et de s'oublier dans un monde textuel. Car, c'est dans cette même prison où il s'engage à défendre les opprimés qu'il découvre un livre à la fois idéal et total, en rupture avec le réel: c'est Ulysse de James Joyce, "le livre idéal dans la mesure où il est [..] inutile [...] impossible à soustraire de la littérature, ancré dans son être, en l'occurrence littéraire" (Ben Jelloun, 1999, Lire).

Ainsi, depuis Harrouda (1973) jusqu'à certains parmi les écrits des années quatrevingt-dix (La Nuit de L'Erreur, Les yeux baissés), le roman, tout en restant - sans aucun doute - engagé moralement et politiquement, devient chez Ben Jelloun un lieu de doute, d'autocontestation et de rupture. L'écriture, qui ne cesse de cultiver sa propre hésitation, aboutit à des formes romanesques qui démultiplient leurs voix narratives, dédoublent et déréalisent leurs personnages, métatextualisent leur fiction littéraire jusqu'à mettre en question leur propre statut ontologique. Le rêve "ulyssien" du livre total revient explicitement en 1999 dans L'Auberge des pauvres - histoire d'un écrivain qui, écrasé par le poids du réel, quitte le Maroc et se réfugie à Naples pour y créer sa propre version d'Ulysse. II aspire à "la fuite dans un monde intérieur, dans un univers de liberté et de création" (Ben Jelloun, 1999: 272) et tente ainsi de réaliser le rêve commun aussi bien à l'auteur lui-même qu'à ses nombreux personnages.

On peut se demander pourtant si L'Auberge des pauvres ne constitue pas une sorte d'adieu au roman qui se repliait subversivement sur sa propre forme. C'est d'autant plus 
possible qu'ayant parcouru son labyrinthe napolitain, après la descente dans le souterrain de ses propres fantasmes, le héros de L'Auberge des pauvres se sent finalement en mesure d'affronter la réalité marocaine.

En effet, à partir des années quatre-vingt-dix, il s'opère, chez Tahar Ben Jelloun, un considérable changement de technique romanesque qui va de pair avec l'homogénéisation thématique. Le roman intertextuel, inspiré par le conte oriental, souvent baroque et surchargé sémantiquement cède peu à peu la place à des textes à la facture beaucoup plus réaliste proche du reportage, dans lesquels le quotidien l'emporte sur le fantasme et le documentaire occupe la place réservée auparavant au métatextuel.

L'homme rompu, Partir et Au pays publiés respectivement en 1994, 2006, 2009, maintenus dans l'esthétique commune, traitent du même sujet: il est question de l'identité marocaine et du rapport des protagonistes à leur pays. Parallèlement à ces représentations romanesques, Tahar Ben Jelloun aborde le problème de l'identité marocaine sur le plan personnel: dans ses chroniques, il redéfinit, au bout de trente ans d'activité littéraire, son statut d'écrivain marocain d'expression française.

Il en résulte une image de l'écrivain chez qui le lien avec ses origines, sa société, sa "tribu" est d'ordre presque vital: en effet, Tahar Ben Jelloun n'envisage pas son activité littéraire en dehors du contexte marocain, son statut d'écrivain restant intrinsèquement lié à son identité marocaine, car comme il l'explique dans la chronique du 27 avril 2008: "être Marocain, c'est s'inscrire dans une histoire. [...] C'est aussi poser les bases d'une identité plurielle" (Ben Jelloun, 2008). Pour Ben Jelloun, dans les dernières décennies, le roman devient donc, par-dessus tout, un terrain d'investigations sociologiques: "[...] en tant qu'écrivain, j'ai la chance d'appartenir à la société marocaine. C'est une chance parce que la réalité marocaine est si complexe, si riche, si contradictoire qu'elle fournit en permanence matière à fiction" (ibid.).

Tout surprenant que cela puisse sembler, l'auteur qui - par ailleurs - doit sa notoriété aux succès des romans (tels que L'Enfant de sable ou La Nuit sacrée) en rupture avec la forme traditionnelle, s'inscrit volontairement, dans les derniers temps, dans "la lignée balzacienne". II renoue avec la conception du roman qui témoigne de son époque en faisant concurrence à l'état civil: "Si nous considérons qu'un écrivain est un témoin de son époque, qu'il fouille la société et ses strates, qu'il fait à sa manière de l'archéologie, le Maroc est un sujet inépuisable. II suffit d'être là, de circuler, d'écouter, d'observer" (ibid.). D'une certaine manière, la "paternité" de Balzac, est déclarée par Ben Jelloun lui-même dans la chronique Suis-je un écrivain arabe du 28 novembre 2004, où il s'explique sur ses investigations sociologiques qui n'ont jamais dépassé le cadre strictement marocain: "[...] la France a assez d'écrivain de toutes sortes! Elle n'a pas besoin d'un Marocain pour la fouiller au sens où Balzac définissait le roman" (Ben Jelloun, 2004). 
II ne faut pas ignorer que l'évolution du romanesque chez Ben Jelloun, qui va dans le sens d'un ancrage plus solide dans le réel, inséparable d'un dépouillement formel, peut être considérée comme une illustration quasi exemplaire des transformations du statut de la littérature annoncées par quarante-quatre auteurs (y compris Tahar Ben Jelloun évidemment) dans le manifeste Pour une littérature - monde en français - paru initialement dans le Monde, le 16 mars 2007. Avec L'homme rompu, Au pays, Sur ma mère, Partir, il s'opère sans aucun doute dans l'écriture benjellounienne ce fameux "retour du monde", "le grand absent de la littérature" et avec lui "la réapparition du [...] sujet, du sens, de l'histoire et du référent". Ayant rompu avec le terrorisme du métatexte, Tahar Ben Jelloun (et il n'est pas le seul parmi les signataires du manifeste) "réapprend à marcher" et "retrouve les voies du monde" pour revenir aux sources de la littérature référentielle.

L'Homme rompu, Partir et Au pays abordent la question de l'identité marocaine, en définissant - par le biais des personnages, trois modèles possibles des rapports au pays natal. Ecrits dans un style oscillant entre un roman-témoignage, un reportage ou même une interview, les histoires de Mourad, Azel et Mohammed, représentants de trois générations successives, composent une sorte de trilogie à la fois identitaire et communautaire, puisqu'au centre des réflexions et actions des personnages il y a le Maroc auquel ils s'identifient des manières différentes mais toujours douloureuses.

Le protagoniste de Partir, Azel, jeune diplômé de droit au chômage, rêve, tout comme la majorité des jeunes de son entourage, d'émigrer en Europe dans l'espoir d'une vie meilleure. II appartient à une génération ratée qui, faute de mieux, se trouve confrontée aux trois choix: d'abord l'immigration - clandestine dans la plupart des cas -, ensuite l'entrée soit dans le monde de la corruption (pour ceux qui ont eu la chance de se faire des relations) soit dans celui de la délinquance (accessible aux moins chanceux) et finalement l'adhésion au mouvement de l'intégrisme islamiste (chemin qui reste ouvert aux frustrés et souvent parmi eux à ceux qui ont échoué ailleurs). Azel dresse l'acte d'accusation contre le Maroc qui l'a renié et déshérité; Partir devient ainsi un plaidoyer en faveur des exclus sociaux condamnés à l'errance.

Agé de 20 ans de plus, le protagoniste de L'homme rompu est un père de famille et ingénieur en bâtiment embauché dans le secteur public. Mourad est lui aussi frustré en se percevant comme une île solitaire dans la mer de la corruption... Souffrant du mépris de son entourage qui prend ses principes moraux pour une simple lâcheté, ne supportant plus la pauvreté à laquelle il condamne non seulement lui-même mais aussi ses enfants, il se laisse finalement absorber par la machine politico-corruptrice. Nous assistons alors à une sorte d'initiation du protagoniste qui entre - pour ainsi dire - dans la tribu. Ainsi, ayant abandonné ses principes, Mourad devient traître: il détruit sa propre image d'un Marocain intègre qu'il a pendant des années, obstinément cultivée. 
Finalement Mohammed, héros principal d'Au pays représente la génération des patriarches. Ayant dépassé le seuil de ses soixante ans - l'âge de la retraite, il décide de réaliser le rêve qui ne l'a jamais abandonné durant quarante ans de sa vie de travailleur immigré dans une banlieue parisienne: il revient dans son bled berbère dans le sud marocain. Le motif du retour dans la patrie a permis à l'auteur d'introduire le thème de la confrontation des représentations mythifiées et nostalgiques du Maroc avec la réalité. Mais ce qui l'intéresse encore plus, c'est un véritable fossé identitaire qui sépare les pères maghrébins, installés en France à l'époque des Trente Glorieuses et toujours tournés nostalgiquement vers leurs patries, et leurs enfants nés en France et étant déjà de nationalité française. La famille de Mohammed illustre le paradoxe propre à de nombreux foyers d'origine marocaine où les deux générations vivant sous le même toit ne partagent plus ni la langue, ni la patrie, ni les modèles de vie.

Ce qui frappe dans la représentation des trois protagonistes, c'est d'abord un solide ancrage spatio-temporel de leurs histoires: avec notamment la mention de la mort de Hassan II en juillet 1999, des élections présidentielles françaises de 2002 opposant Chirac à Le Pen, de l'affaire "caricatures de Mohamet" de septembre 2005, des banlieues parisiennes en flamme en octobre 2005 et d'autres événements encore - Ben Jelloun réalise le modèle du roman d'actualité ou celui de la chronique de l'époque.

Mais ce qu'il faut également souligner, c'est le caractère prototypique des protagonistes: fortement représentatives, leurs histoires se déroulent surtout sur le plan collectif. En effet, Mourad, Azel et Mohammed se positionnent toujours par rapport à d'autres personnages appartenant aux mêmes catégories socio-professionnelles. Les héros principaux sont des pivots autour desquels tournent leurs "semblables". Ceux-là, plongés dans le même milieu, fonctionnent comme des variantes des protagonistes, puisqu'ils n'existent dans la diégèse qu'en tant que modèles de comportements concurrentiels aux choix de ces derniers.

Ainsi, Mourad, hésitant entre intégrité et corruptibilité, rencontre ses doubles: Abbas corruptible et sans aucun scrupule et "Grain de sable" toujours honnête. Quant à Mohamed, dans son statut récemment acquis de retraité, il se compare à ses voisins et collègues d'usine, tous ghettoïsés dans la même banlieue, et qui doivent, chacun à sa manière, s'adapter à la vie inactive. Ainsi, à l'arrière plan de l'histoire du protagoniste se tissent une série d'histoires d'ouvriers marocains retraités. Ils fournissent autant de modèles complémentaires à la décision de Mohammed de revenir au pays et qui oscillent notamment entre la plongée dans l'alcoolisme (Bahir) ou l'isolation et la solitude (Hadj Momo).

En effet, dans Au pays, nombreux sont les procédés qui font de Mohammed le porteparole de la première génération marocaine de travailleurs immigrés. D’abord, en imitant la convention du reportage, la narration alterne librement la troisième et la première personne 
pour laisser la parole au protagoniste. Or, celui-ci en profite souvent pour introduire la voix plurielle "nous" en signe d'identification avec le groupe. De cette manière, la rétrospection de sa vie depuis son départ du bled en 1960 jusqu'à son retour effectué en 2005 devient une véritable chronique de l'immigration qui contient quelques étapes majeures:

- Départ massif autorisé par le makzen (incarnation du pouvoir central et chef incontestable de la communauté): "mais, nous nous avons le makzen [...] qui nous commande" (Ben Jelloun, 2009: 70). II ne s'agit en aucun cas de rupture avec le pays, l'identité marocaine des émigrés n'étant nullement mise en question.

- Installation provisoire en France et sentiment de la non appartenance à la société occidentale, ghettoïsation: "on ne parlait pas de nous; nous étions dans des cités de transit puis nous n'allions presque jamais en ville" (ibid.: 69).

- Montée des racismes anti-arabes, émergence de Le Pen: "Qu'avons-nous fait de si terrible pour être suspectés, parfois maltraités dans la rue?". II s'en ensuit une radicalisation du ton qui reprend en écho les idées de L'Hospitalité française: "Que faire? Disparaître! Ne plus exister, devenir transparents, tout en continuant à bosser: être là, être utiles, efficaces puis ne pas se montrer, ne pas faire d'enfants, ne pas cuisiner avec nos épices qui dégagent des odeurs dérangeantes!" (ibid.: 68-9).

Le lecteur est ainsi amené à lire l'histoire de l'immigration de Mahommed en continuité avec La Réclusion solitaire: comme le confirme Tahar Ben Jelloun, les deux romans (quoique maintenus dans des styles bien différents) mettent en scène le même personnage qui résume à lui seul les problèmes existentiels de toute une génération ${ }^{1}$. "Allez Mokhamad, bienvenu" - cette salutation qu'un employé adresse au protagoniste à son arrivée à la cité de transit met l'accent - non sans une triste ironie - sur la typicité de son histoire puisque, pour le français qui le reçoit, son prénom n'a qu'une valeur généralisante de nom commun: "[...] il appelait tous les immigrés Mokhamad" (ibid.: 113).

De manière analogue dans Partir, le statut communautaire du protagoniste est tout à fait évident, car, à l'arrière-plan de sa trame, il se brosse une quinzaine de portraits parallèles de jeunes immigrés qui se recrutent parmi ses proches, amis, collègues. Ecrites tantôt à la troisième tantôt à la première personne, ces histoires se fondent toutes dans une sorte de voix plurielle - le "nous" collectif d'Azel qui constate notamment: "Nous sommes

\footnotetext{
1 "Cet homme est celui de La Réclusion solitaire, qui a vieilli et a eu femme et enfants. Le livre clôt un cycle commencé il y a 32 ans." Cf. Tahar Ben Jelloun: Les immigrés sont des invités qui ne veulent déranger personne. http://www.magazine-litteraire.com/content/recherche/article?id=13115
} 
nombreux à être jeunes avec un horizon bouché, pourri, rien à l'horizon" (Ben Jelloun, 2006: 184). Et sa voix est d'autant plus grave, elle résonne d'autant plus fort que, dès son départ du Maroc, le héros adresse des lettres à sa patrie, en utilisant toujours comme terme d'entrée, deux invocations: "Cher Pays" ou "Mon Cher Pays".

La personnification du pays est un procédé rhétorique très efficace. Quand l'auteur attribue au protagoniste le statut du scripteur, c'est comme s'il lui donnait le plein pouvoir énonciatif: en effet, dans ces quatre lettres, Azel passe aisément d'une tonalité à l'autre en se rapprochant dans son style tantôt d'un discours politique - comme dans une interrogation: "peut-on être raciste contre son propre camp" (ibid::106), tantôt d'une confidence - "ô mon pays, si tu savais ce que je suis devenu" (ibid.: 107), tantôt d'une lettre d'amour - "me voici loin de toi et déjà quelque chose de toi me manque" (ibid.: 93), tantôt finalement d'une prière - comme dans l'extrait ci-dessous: "ô, mon pays, ma volonté contrariée, mon désir brûlé, mon regret principal, mon soleil, ma tristesse [...]" ou encore "débarrasse-nous de ces voyous qui te saignent" (ibid:: 90) Cette diversité énonciative rend surtout compte de la polyvalence sémantique du concept du pays. "Pays" et "Maroc", ces deux termes, dont la récurrence dans la dynamique textuelle peut sembler obsédante, deviennent des mots de passe pour accéder à la problématique complexe; ils fonctionnent comme des métonymies capables d'absorber des thèmes variés.

Multiple et plein de contrastes, le Maroc invite Azel à des prises de position ambivalentes, ses sentiments allant d'une rage contre la patrie qui l'a rejeté à un mal de pays très fort.

Le Maroc éveillant sa nostalgie est de nature maternelle: métonymiquement, il se renferme dans le souvenir de la mère analphabète, Lalla Zohra, récitant le Coran. Sur ce point, Azel ressemble au protagoniste d'Au pays qui trouve dans l'islam sa patrie, sa culture, son identité et qui n'a jamais distendu les liens avec sa famille et son bled berbère (sa tribu). Comme Mohammed, dont la "marocanité" a le goût du miel et l'arôme de l'huile d'olive, Azel associe l'idée de sa patrie au bonheur familial, aux odeurs de la cuisine. Ainsi, en recevant en Espagne sa soeur Kenza munie de spécialités culinaires préparées par leur mère, c'est son Maroc idyllique et enfantin qu'il retrouve: "Le Maroc débarquait en Espagne avec des tajines de poulet aux olives et citron confit, des pastilles de caille, des cornes de gazelle, des gâteaux du miel pour le ramadan [...] de l'encens et un dossier à remplir sur lequel était écrit Lalla Zohra" (ibid.: 172).

Ainsi représenté, l'amour du pays correspond tout à fait aux représentations traditionnelles: visiblement, Tahar Ben Jelloun, sans avoir peur de tomber dans la banalité, cherche à être sincère en décrivant avec simplicité et modestie et sans aucune prétention d'originalité, les émotions de ses compatriotes et surtout le mal de vivre en exil: "[...] partout où vous irez, quel que soit le travail que vous ferez, une chose est sûre: le Maroc ne vous 
lâchera jamais [...] il vous collera à la peau [...]“ (ibid.: 106). Ce présage donné à Mohammed et ses collègues en voyage par un ancien émigré répond en écho aux paroles de Lalla Zohra qui prédit à sa fille: "tu auras beau partir, ton pays te manquera toujours. Le Maroc, on s'y attache très fort [...] il attache, dans le vrai sens du mot, comme une poêle [...]" (ibid.: 97).

Or, à l'opposé d'un Maroc idyllique, maternel et émouvant il y a un Maroc néfaste, destructeur. Ce Maroc-ci a le visage d'un homme corrompu, d'un chef de mafia (Al Afia) ou d'un pèlerin hypocrite, un "haj" qui part en pèlerinage avec de l'argent sale... car comme le commente Azel: "soigner les apparences et faire des cochonneries en douce, c'est ça, le Maroc qui m'énerve" (ibid:: 97). A ses côtés, ou plutôt sur le corps de ce pays malsain et opportuniste se greffe - comme un parasite - et ne cesse de grandir le Maroc des extrémistes et des fanatiques, c'est - dans le discours propagandiste "[...] un Maroc rendu à l'islam, à la probité, à l'intégrité et à la justice" (ibid.: 26).

A ce propos, nous tenons à souligner qu'Au pays et L'homme rompu constituent deux études perspicaces et complexes (car appuyées sur plusieurs exemples) du lien entre, d'un côté, la pauvreté et la corruption et, de l'autre, sa plus logique conséquence - l'extrémisme: l'islam dans sa variante politisée et populiste, où se canalisent frustration, manque de perspectives et obscurantisme.

Métonymie des problèmes sociaux, le concept du Maroc subit aussi, chez Tahar Ben Jelloun, une série de personnifications. Kenza, soeur d'Azel, apprend de Miguel - qui représente sur ce point les idées de l'auteur lui-même: "tu es le Maroc de demain, ce sont les femmes qui feront bouger ce pays, elles sont formidables" (ibid.: 173).

Or, à la différence de Kenza, qui, pleine d'espoir à la mort du roi Hassan II, décide de revenir au pays, d'autres incarnations du Maroc ne sont pas aussi catégoriquement positives. C'est notamment Mourad qui constate: “[...] je m'identifie au pays et me dis que si moi, j'émerge, lui aussi sera sauvé" (Ben Jelloun, 1994: 73). Ce sous-directeur de la planification, du progrès et de la perspective (titre cruellement ironique dans son cas) a basé toute son éthique sur son intégrité, à contre-courant de tout ceux qui (en cultivant un art hypocrite de l'euphémisme) expliquent la corruption comme "une économie parallèle, un impôt supplémentaire, un consensus national, une compensation, une course à l'équilibre, une souplesse" (Ben Jelloun, 1994: 33). Hélas, même lui se laisse finalement vaincre: symboliquement, il adhère au système de Zarathoustra et remplace Sartre par Nietzsche. Avec le motif de la chute de L'Etre et le Néant, "ce pavé que personne n'irait lire" (Ben Jelloun, 1994: 74) et qui - pour cette raison même - ne servira à Mourad que d'une cachette habile pour l'argent sale; avec la mort des principes de - paraît-on - l'un des derniers survivants de l'honnêteté, L'homme rompu, histoire emblématique et exemplaire à rebours devient la parabole d'un naufrage économique et moral du Maroc. 
La fonction symbolique du protagoniste de Partir va encore plus loin, puisque son nom, Azz El Arab (dont dérive le diminutif Azel) signifie "la gloire des Arabes". Ce prénom est sarcastique, car, comme le constate le héros lui-même: "Malheureusement, le monde arabe est aujourd'hui en bien mauvais état, moi aussi d'ailleurs" (Ben Jelloun, 2006: 58). Incarnation d'une certaine "arabité" donc, ce personnage permet à l'auteur de réviser la notion, tout en rendant compte des pièges qu'elle semble inclure. Au fur et à mesure que son histoire progresse, il s'avère qu'Azel n'est pas seulement une victime du marasme où plonge son pays. Car instruit et moderne au début de son parcours identitaire, il se laisse petit à petit gagner par des clichés. En effet, plus grand est l'échec d'Azel et plus difficile sa condition matérielle et morale, moins lucide devient sa façon de voir la réalité. Aussi n'échappe-t-il ni au racisme - “j'aime pas les Turcs, j'aime pas leur langue, j'aime pas leur regard" (ibid:: 218) -, ni à la superstition que lui a inculquée sa mère: chassé par son protecteur Miguel, il jette la responsabilité sur la domestique de ce dernier, Carmen: "elle a dû faire marcher ses sorciers et sorcières [...] même les Européens ont recours à ce genre des choses" (ibid.: 219). Et finalement, dans l'épisode de la soirée marocaine - "l'Orient en rose", le protagoniste étale, devant une bohème barcelonaise blasée - une image simpliste et stéréotypée d'un Maroc qui, pour se plier aux attentes des touristes, vend son côté folklorique.

Ainsi, Azel ne fait que signaler les motifs organisateurs des deux recueils de nouvelles de Tahar Ben Jelloun Les premières amours sont toujours les dernières et surtout Amours sorcières qui illustrent le conflit entre la tradition et la modernité dans le Maroc actuel.

La mort d'Azel est une conséquence inéluctable de sa chute progressive. Passé dans la clandestinité, sans ressources, enrôlé finalement par les frères musulmans, avant de devenir l'espion de la police espagnole, ce personnage quasi picaresque à la fin, se laisse brutalement assassiner par des fanatiques musulmans le jour de la fête du sacrifice: "Azel était par terre, la gorge tranchée, la tête dans une flaque de sang. Comme un mouton de L'Aïd-el-Kébir, les Frères l'avaient égorgé" (ibid:: 306). Bestiale, privée de dignité, comme issue directement d'un roman policier, cette mort, en même temps sacrificielle, scelle un échec presque communautaire.

L'homme rompu, Partir, Au pays fournissent trois portraits cruels et véridiques du Maroc actuel. Engagé surtout moralement, Tahar Ben Jelloun porte un regard froid, lucide, clinique même sur la société et ses maux en fournissant une série de portraits à la dimension surtout collective. Fidèle à la conception de l'"écrivain public ", il prête ses romans à l'action des voix polyphoniques; il s'efface derrière ses personnages, les laisse surtout parler et, parfois aussi, agir. Aussi assistons-nous à la réalisation du modèle d'un roman le plus démocratique et en même temps le moins romanesque possible. 
Or, dans ces trois romans, il existe des moments bien particuliers où Tahar Ben Jelloun renonce à son attitude impersonnelle, à sa transparence: en ouvrant à ses personnages trois territoires de la liberté il leur prête son propre rêve, inscrit dans son projet d'écriture et qui consiste à rompre avec le réel devenu insupportable. Car, dans les parties finales des romans, le vraisemblable est concurrencé par l'insolite; l'onirisme entre de pleinpied dans l'univers hyperréaliste. Trois fantasmes, trois chutes dans le délire - en contraste avec l'esthétique réaliste dominante dans les romans - illustrent l'idée de la fonction consolatrice du rêve, de la folie et de la littérature en fin de compte.

D'abord, dans L'Homme rompu, la symbiose de la machine à écrire Olivetti et du dictionnaire français Larousse qui donne comme fruit des textes surréalistes apaise et réconforte Mourad. Puis, le dernier chapitre de Partir constitue un épilogue présentant des émigrés qui, ayant choisi le seul chemin possible pour échapper à la lourdeur du réel, partent pour le monde de la fiction, sur un bateau qui rappelle la Nef des fous: ils se déréalisent et se textualisent sous l'égide d'ailleurs de Moha-le-fou Moha-le-sage, leur initiateur au délire. Finalement dans le dénouement d'Au pays, nous retrouvons Mohamed qui en délirant "s'arborise" dans sa maison nouvellement construite: il s'enracine et s'affaisse dans le sol pour cesser d'exister, sa mort renvoyant directement au réalisme magique propre notamment au Cent ans de solitude de Gabriel García Márquez. Occupant une place modeste, symbolique dans l'économie des romans, ces trois motifs, absolument inattendus et en rupture formelle avec les chapitres précédents, jettent une lumière nouvelle sur les personnages. Car, en les arrachant à leur existence, triviale, en leur octroyant, ne serait-ce que pour un bref moment même, une sorte de statut d'exception, Tahar Ben Jelloun essaie peut-être de les réhabiliter ou même, en dépit de leurs défaillances, de leur rendre hommage. Ainsi, dans les dernières années, le roman benjellounien - en continuité avec les écrits précédents - constitue un espace de la compassion et de la solidarité. 


\section{Bibliographie}

BEN JELLOUN, Tahar (1973). Harrouda. Paris: Denoël.

BEN JELLOUN, Tahar (1983). L'Ecrivain public. Paris: Seuil.

BEN JELLOUN, Tahar (1994). L'Homme rompu. Paris: Seuil.

BEN JELLOUN, Tahar (1997). La Nuit de l'Erreur. Paris: Seuil.

BEN JELLOUN, Tahar (1999). L'Auberge des pauvres. Paris: Seuil.

BEN JELLOUN, Tahar (2006). Partir. Paris: Gallimard.

BEN JELLOUN, Tahar (2009). Au Pays. Paris: Seuil.

BEN JELLOUN, Tahar (2004). Suis-je un écrivain arabe? Chronique du 28 novembre 2004,

$<$ http://www.taharbenjelloun.org/chroniques.php?menuimg=3\&type_texte=0\&id_chronique=9 [consulté le 30 mars 2010].

BEN JELLOUN, Tahar (2008). Etre Marocain. Comment se définir en tant que Marocain. Chronique du 24 avril 2008, $<$ http://www.taharbenjelloun.org/chroniques.php?menuimg=3\&type_texte=0\&id_chronique=109> [consulté le 30 mars 2010].

Tahar Ben Jelloun. Propos recueillis par Catherine Argand. Entretien."Lire", mars 1999, <http:/ /www. taharbenjelloun.org/chroniques.php?menuimg=4\&type_texte=2> [consulté le 30 mars 2010].

BEN JELLOUN, Tahar (2009) Les immigrés sont des invités qui ne veulent déranger personne. $<$ http://www.magazine-litteraire.com/content/recherche/article?id=13115> [consulté le 30 mars 2010].

Pour une littérature-monde en français. Le Monde, le 16 Mars 2007. Par Muriel Barber..., <http: //www.lemonde.fr/cgibin/ACHATS/acheter.cgi?offre=ARCHIVES\&type_item=ART_ARCH_30J\&obj et_id=980496> [consulté le 30 mars 2010]. 